\title{
PARVALBUMIN IMMUNOREACTIVITY IN THE RETICULAR THALAMIC NUCLEUS OF DEVELOPING RATS
}

\author{
Акıко SETO-OHSHIMA ${ }^{1}$, Eiko AOKI ${ }^{1}$, Reiji SEMBA $^{1}$, \\ Piers C. EMSON ${ }^{2}$ and Glaus W. HEIZMANN ${ }^{3}$
Institute for Developmental Research ${ }^{1}$, Aichi Prefectural Colony, Aichi 480-03, $M R C$ Group ${ }^{2}$, Institute of Animal Physiology and Genetics Research, Cambridge CB2 4AT, U. K. and Department of Pediatrics ${ }^{3}$, Division of Clinical Chemistry, University of Zürich, 8032 Zürich, Switzerland

Received for publication March 27, 1989 and in revised form April 17, 1989

\begin{abstract}
Developmental changes in the distribution of parvalbumin immunoreactivity in the rat reticular thalamic nucleus from embryonic day 18 to adult were studied using immunohistochemistry. Neurons containing parvalbumin immunoreactivity in their cell somata were first detected at embryonic day 18. By embryonic day 20, many cells extended parvalbumin-positive fibers into the ventral thalamic nucleus. During development of the ventral thalamic nucleus, these fibers increased in number and thickness and ran in various directions. By postnatal day 16, these fibers became thinner and less well-stained. Neurons in the reticular thalamic nucleus extended their parvalbumin-positive axons into other nuclei of the thalamus, but in these other nuclei, parvalbumin-positive terminal and fibers developed primarily postnatally. In the reticular thalamic nucleus, the distribution of GABA-like immunoreactivity was very similar to that of parvalbumin at embryonic day 20 with cells extending fibers which contained immunoreactivity for GABA into the ventral thalamic nucleus. Thus parvalbumin immunoreactivity appears in the neurons of the reticular thalamic nucleus early in development of this nucleus, first in cell somata of neurons, then in their axons and dendrites. Apart from the ventral thalamic nucleus, the innervation by parvalbumin containing neurons in the reticular thalamic nucleus of the rest of the thalamus, showed a variable developmental pattern with different nuclei being innervated at different times, presumably reflecting the separate development of individual thalamic nuclei.
\end{abstract}

The reticular thalamic nucleus (RTN) is a nucleus with a characteristic shell type structure which surrounds the lateral, anterior superior and anterior inferior surfaces of the thalamus. Neurons of RTN project their axons mostly into other thalamic nuclei $(9,13,15,17,18)$. They are believed to contain the inhibitory neurotransmitter, gamma-aminobutyric acid (GABA) (8), and may play a role in the modulation of thalamocortical activities $(13,18)$.

The reticular thalamic neurons also contain high levels of the calcium-binding

Correspondence should be sent to Dr. Akiko Seto-Ohshima, Institute for Developmental Research, Aichi Prefectural Colony, Kamiya-cho 713-8, Kasugai, Aichi 480-03, Japan. 
protein, parvalbumin (PV) $(4,6,7)$. These findings were confirmed by "in situ" hybridization using a radiolabelled rat parvalbumin cDNA probe (19). In the brain, $\mathrm{PV}$ is present in a distinct sub-population of neurons $(4,7)$ especially in the fast firing GABAergic neurons such as RTN neurons (11) and is thought to participate in neuronal function by its capacity to bind or buffer calcium-ions in the micromolar range.

In this study, the organization of RTN structure was followed during rat brain development using antibodies raised against PV as well as GABA. The aim of this work was to investigate at which time point the reticular thalamic nucleus system was organized.

\section{MATERIALS AND METHODS}

Antibodies: Specific antiserum against rat muscle PV (10) and a specific antibody against GABA conjugated to rabbit serum albumin (1) were used in this study. Both antisera were raised in rabbits.

Tissue preparation: Pregnant Sparague-Dawley rats were anaesthetized with urethane $(1 \mathrm{~g} / \mathrm{kg}$ ). Embryos (embryonic day (E) 18 and 20) were quickly removed and brains were fixed in $4 \%$ paraformaldehyde in $0.1 \mathrm{M}$ phosphate buffer, $\mathrm{pH} 7.4(\mathrm{P}$ fixative) at $4^{\circ} \mathrm{C}$ for more than $24 \mathrm{hr}$ or in the fixative for GABA immunohistochemistry ( $\mathrm{G}$ fixative: 1\% sucrose, 10\% formalin, 1\% glutaraldehyde, $0.2 \%$ picric acid in $0.1 \mathrm{M}$ sodium acetate buffer, $\mathrm{pH} 6.0$ ) for $3 \mathrm{hr}$ at room temperature. After fixation, they were washed with cold $0.1 \mathrm{M}$ phosphate buffered saline (PBS). The day on which a vaginal plug was detected was designated as being gestational day 0 . Postnatal animals (P0-the day of birth, $\mathrm{Pl}, 3,7,11,16,33,60)$ were perfused with saline followed by $\mathrm{P}$ or $\mathrm{G}$ fixative under anaesthesia with chloral hydrate $(350 \mathrm{mg} / \mathrm{kg}$ body weight) and the brains removed were treated in the same way as embryos. The amount of fixative used ranged from 20 to $100 \mathrm{ml}$ according to the body weight. Fixation time with $\mathrm{G}$ fixative varied from 3 to $16 \mathrm{hr}$ depending on the size of brain. Some brains which were fixed in Bouin's fixative for $3 \mathrm{hr}$ or in $\mathrm{P}$ or $G$ fixative were embedded in paraffin (Histosec; Merck) according to routine methods after dehydration with graded ethanol. The sections were stained with haematoxylin and eosin to see the orientation.

Immunohistochemistry: Freezing-microtome sections $(30-50 \mu \mathrm{m})$ were made after transferring brains into $10 \%$ sucrose in PBS for cryoprotection. They were washed in cold PBS, pretreated with 8\% normal goat serum (NGS) in PBS for $1 \mathrm{hr}$ and incbated with primary antibody (PV antibody, $1: 8000$ in $1 \%$ NGS, $0.003 \%$ Triton X-100, $0.02 \% \mathrm{NaN}_{3}$. GABA antibody, $1: 400$ and $1: 1000$ in the same solution without Triton $\mathrm{X}-100$ ) for 2 day at $4^{\circ} \mathrm{C}$. The localization of antigen-antibody complex was visualized with the ABC method using a VECTASTAIN kit (Vector Lab.) or by incubation with peroxidase-labeled goat anti-rabbit IgG (dilution, $1: 100$ to $1: 150$, Cappel laboratories, West Chester, U.S.A.) followed by peroxidase reaction $(0.1 \%$ $3,3^{\prime}$-diaminobenzidine and $0.045 \%$ hydrogen peroxide).

Absorption with individual antigen $\left(10^{-5} \mathrm{M}\right)$ and use of nonimmune rabbit serum instead of specific antibodies revealed that all the staining observed was specific. 


\section{RESULTS}

Changes in the distribution of PV immunoreactivity in the developing RTN were studied using floating sections from brains fixed with $P$ fixative. G fixative also could be used to detect PV immunoreactivity. Paraffin sections were also tested but required high concentration of PV antibody to visualize PV immunoreactivity.

At E18, PV immunoreactivity was only localized in restricted areas of the rat brain. Some fiber systems in the hindbrain including oculomotor fibers and neurons in the mesencephalic trigemial nucleus showed strong PV immnoreactivity. However, in RTN, there was only weak but heterogeneous immunoreactivity in cell somata of neurons at this age. A few neurons situated at the ventral region of RTN had short immunopositive processes extending horizontally. By E20, cell somata of neurons of RTN had acquired more immunoreactivity. In addition, long straight fiber-like structures extended from some cells of RTN into the ventral thalamic nucleus (Fig. 1). With higher magnification, these structures were found to be made up of many PV-positive dots accompanied with more weakly stained dots around them. In horizontal sections at the level where PV-positive fiber-like structures were found in sagittal sections, such fiber-like structures extended like a fan into the ventral thalamus. In the anterior region of RTN, a group of neurons in the internal capsule were PV-positive, and between these neurons and RTN neurons, and in the more ventral region, short fibers containing PV-positive dots were found.

On the day of birth (P0), most parts of RTN except for the most dorsal part had neurons which extended long fiber-like structures into almost all the ventral thalamic nucleus as well as some parts of the posterior nuclear group. A number of dots was also found beyond the superior thalamic radiation. At P3, PV-positive fiber-like structures increased in number (Figs. 2, 3a). Sometimes fibers had continuous staining with or without immunopositive dots. Most of them ran in an anteroposterior direction but a few ran in a random way into the ventral thalamic nucleus. From the dorsal part of RTN, short immunopositive fibers were found to extend to the lateral thalamic nucleus where a low density of immunopositive dots was also distributed. In the dorsal and medial geniculate nucleus, immunopositive dots were also found. At P7, the fibers extending into the ventral thalamic nucleus became thicker and many segments ran crossing each others (Fig. 3b). The density of PV-positive dots in the geniculate nucleus increased. In the anterodorsal thalamic nucleus, a small number of dots appeared at P7 and at P11, they became very dense and immunopositive fibers were found among them (Figs. 4a, b). This nucleus seemed to be innervated by segmented fiber bundles running from the dorsal part of RTN (Figs. 4a, b, c). In the

FIG. 1. PV immunoreactivity in RTN at E20. At E20, RTN neurons acquire clear immunoreactivity which is weak at E18. At this age, some neurons extend PV-positive fibers which are made of PV-positive dots towards the ventral thalamic nucleus in sagittal section. Blood cells show pseudo-peroxidase reaction product (arrow) (a). Diagram of PV immunoreactivity in RTN and surrounding areas which correspond to the figure (a) is illustrated (b). a $\times 68$

Fig. 2. PV immunoreactivity in RTN at P3. At P3, some thin fibers show continuous staining (arrow) and these fibers and PV-positive dots which are arranged into lines cover most of the ventral thalamic nucleus. There are occasionally a few PV-positive neurons in the thalamus (arrowhead). Some blood cells remaining show pseudo-positive reaction. Diagram of PV immunoreactivity which corresponds to the figure (a) is illustrated (b). a $\times 54$. 

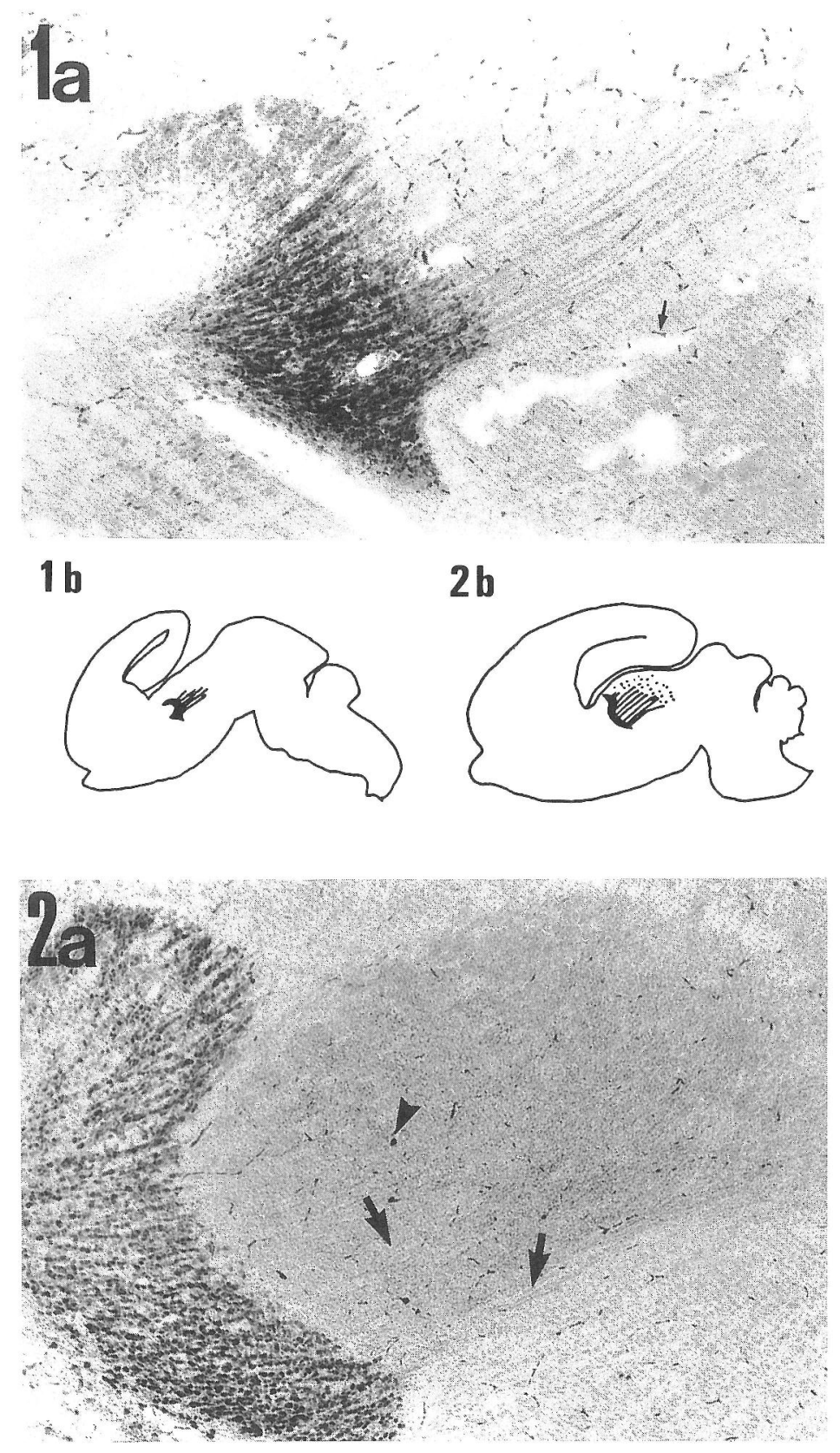

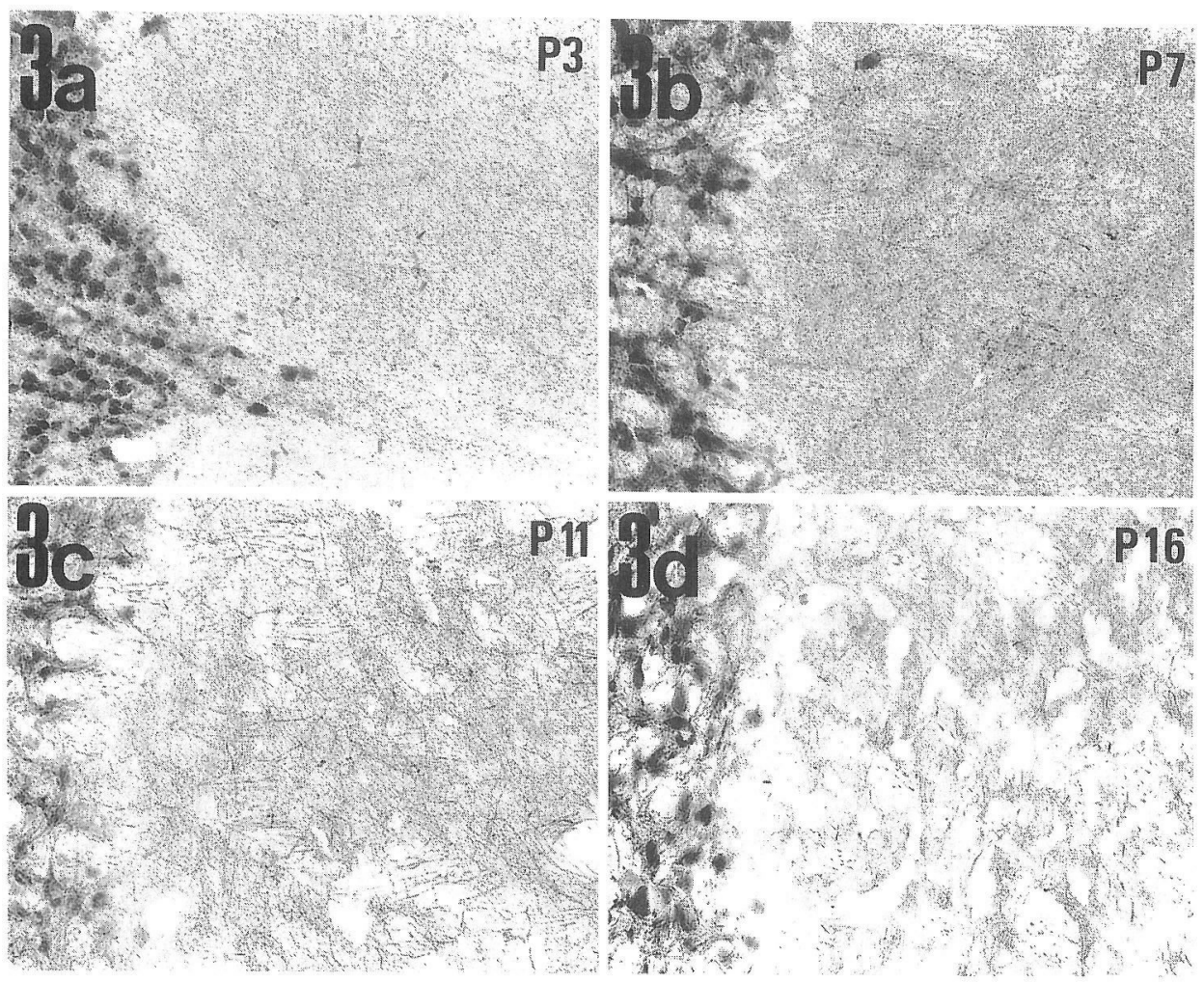

FIG. 3. Changes of PV immunostaining in RTN and the ventral thalamic nucleus in sagittal sections during development. At P3, PV-positive dots which are arranged into lines and PV-positive thin fibers appear mostly to run in anteroposterior direction (a). At P7, the fibers extending from RTN become thicker and not a few fiber segments are found to run to cross each other (b). At P11, still more thick PV-positive fibers are found (c), however, they seem to become thinner at P16 as like those of adult (d). Within RTN, there are PV-positive dots at P3 (a). At P7, clear staining is found in fibers extending from neurons which make a network within this nucleus (b). At P11, the profile is similar to that of P7 (c), and at P16, they become PV-positive segments which appear around RTN neurons as those of adult (d). a, b, c and d $\times 120$

more lateral part, long PV-positive fibers ran beyond the ventral thalamic nucleus into the medial geniculate nucleus (Figs. 4d, e). In the ventral thalamic nucleus, it became difficult to follow the fibers but segments of rather thick fibers ran in variable directions seemingly forming a complex network at P11 (Fig. 3c). At P16, however, these thick fibers seemed to become rarer and segments of thinner fibers ran in every direction as in the adults (Fig. 3d). At this age, several other areas near RTN also contained PV-positive neurons and fibers. In the fiber bundles of the radiatio intermedia thalami, many fibers showed PV-immunoreactivity. Medial lemniscus also gained PV-immunoreactivity, which made it difficult to follow genuine RTN fibers in the ventral portion of ventral thalamic nucleus.

Within RTN, numerous immunopositive dots were found between neurons at P1 but discrete processes extending from RTN neurons seemed to develop at P3 (Fig. 


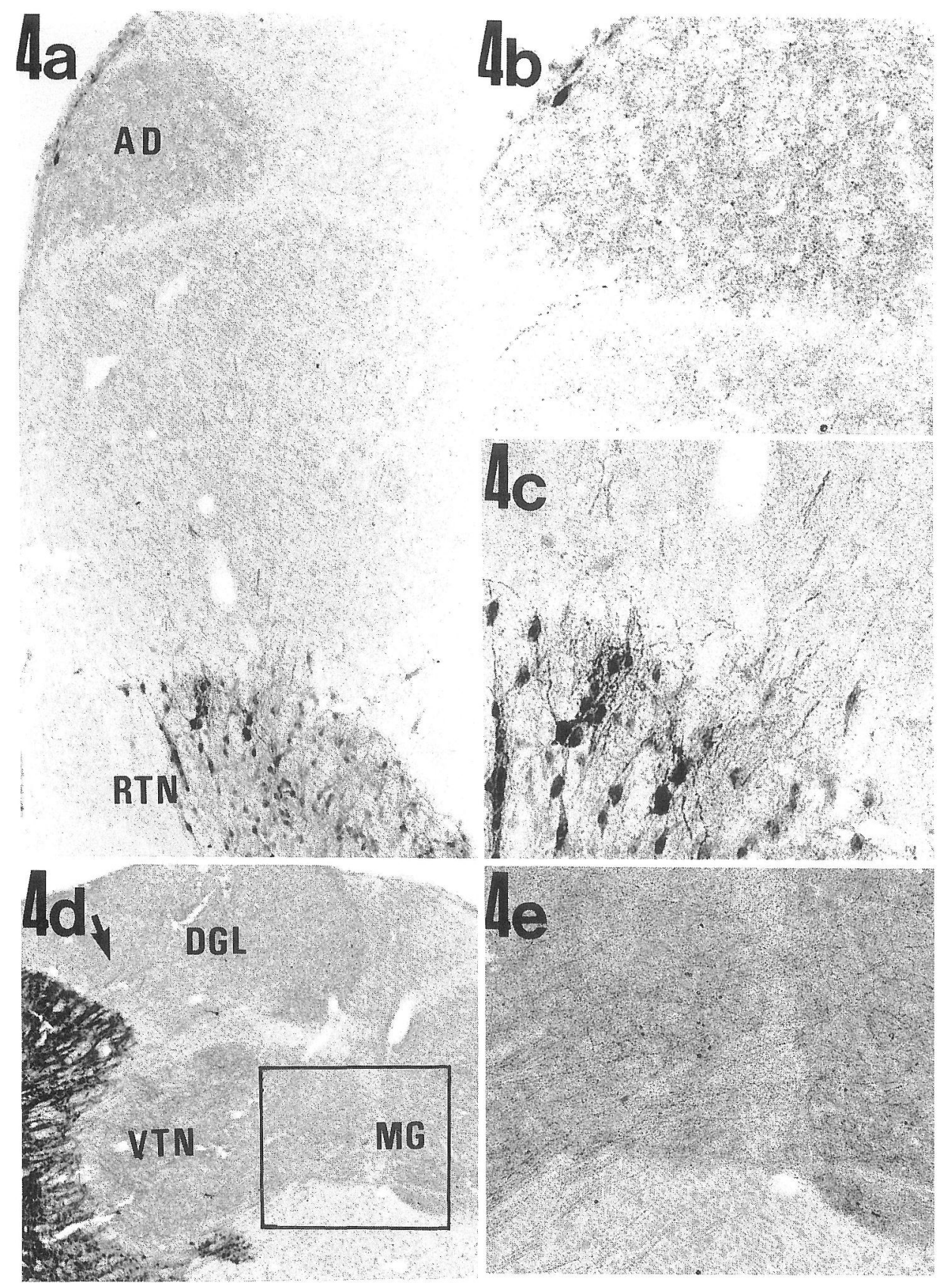

FIG. 4. Distribution of PV immunoreactivity around RTN at P11. The neurons in the dorsal part of RTN send their PV-positive fibers to the anterodorsal thalamic nucleus (AD) (a). Fiber segments with PV immunoreactivity are found to extend from RTN neurons (c) to enter into AD where PV-dots exist around cell somata (b). In a more lateral section, PV positive fibers extend from RTN to the dorsal lateral geniculate nucleus (DGL) (arrow) (d). The box in the (d) indicates clear PV-positive fibers between the ventral thalamic nucleus (VTN) and the medial geniculate nucleus (MG) at high magnification (e). a $\times 77, \mathrm{~b}$ and $\mathrm{c} \times 170, \mathrm{~d} \times 34, \mathrm{e} \times 170$ 

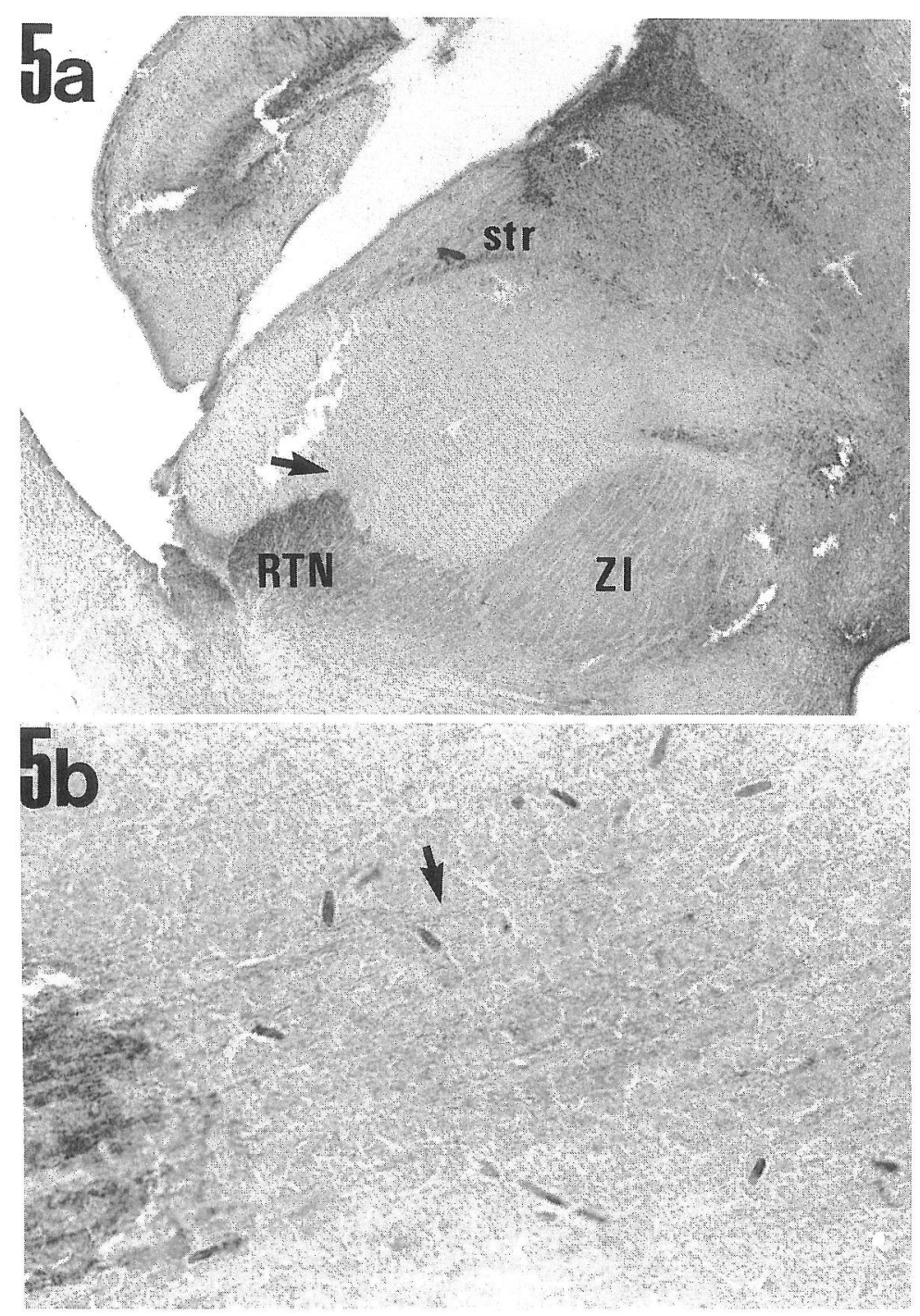

FIG. 5. Distribution of GABA-like immunoreactivity at E20. Several neurons of RTN have GABA-like immunoreactivity positive thin fibers like those found with PV immunoreactivity (arrow). However, GABA-like immunoreactivity is found not only in RTN but also in Zona Incerta (ZI) and superior thalamic radiation (str). They surround the mostly immunonegative thalamus (a). High magnification of GABA-immunoreactivity positive fibers which extend from RTN neurons is shown (arrow). Blood cells show pseudo-peroxidase reaction product (b). a $\times 34$, b $\times 170$ 
3a). At P7, such processes were well developed and made up a fiber network around neurons (Fig. 3b). At P16, these fibers showed the appearance of immunoreactive segments as in the adult (Fig. 3d).

The developmental profile of GABA-like immunoreactivity in RTN was studied with floating sections of the materials fixed with G fixative. As E20, RTN neurons showed GABA-like immunoreactivity, though the intensity of staining varied between cells. Some neurons had immunopositive thin fibers and their profile was very similar to that of PV at the same age, though the former was found in more areas around RTN (Fig. 5). Thereafter the GABA-like immunoreactivity containing fibers extending from RTN neurons increased in number and settled into the final adult innervation pattern.

\section{DISCUSSION}

Numerous fiber systems including the thalamo-cortical, cortico-thalamic and caudato-thalamic fibers transverse the RTN with their collaterals ending within this nucleus. RTN neurons themselves send their axons into the main bulk of the thalamus, where again many fiber systems cross over. Thus, the RTN and its surrounding areas have a rather complex organization. Because of the difficulties of following genuine RTN fiber systems, in such complex situation, the structure of the RTN of adult experimental animals has been studied by using the Golgi method (18), by anterograde axonal degeneration (13), by retrograde transport of horseradish peroxidase, or radiolabelled amino acids (9) or by fluorescent tracers (20). However, despite these detailed studies, there is, to date, little knowledge about the development of the RTN.

As PV is present only in a restricted population of neurons in the brain especially in early stage of the development (14), its early expression in these nuclei (such as RTN) makes it a useful tool with which to follow their development. This study shows that PV immunoreactivity in RTN neurons appears immediately after the formation of this nucleus which occurs at E17 (5). Then immediately after this the neurons extended PV-positive fibers into the thalamus. We cannot exclude the possibility completely that the neurons extended their axons without PV and then PV staining develops. However, a very similar profile to that of PV was obtained with GABA, another cellular marker of RTN neurons. GABA immunoreactivity developed at E20, when some cells extended PV-(and/or GABA-) positive fibers into the ventral thalamic nucleus. So, it is likely that development of RTN can be followed using PV antibody though very fine processes may escape observation.

It is an interesting observation that early developing RTN neurons sent their PVpositive fibers straight into the thalamus, which may form the basis of the topological connections between RTN and the thalamus of adult rat. However, in the adult, such topology is not strict (9) and this may be partly due to the later ramification of the later occurring axons. It is also possible that other early developing thalamo-cortical fibers or some other systems may guide the growth of the PV-positive fibers from the RTN into the thalamus, but this has not been established $(2,12,13)$.

PV-positive fibers from RTN to other parts of the thalamus seem to develop with different time schedules, though in some cases, the presence of weakly staining PVpositive dots, instead of $\mathrm{PV}$-positive fibers make the determination of exact time point 
of extension of RTN axons difficult to determine. Under the conditions of these experiments, many PV-positive dots were found in the thalamus as well as in RTN itself during the development of RTN. Some of these immunopositive dots were arranged in lines and later these presumptive fibers developed continuous fiber staining. Other dots surrounded the neurons. They may be synapses but the characterization of these dots and also of those found in developing fibers remain to be established.

$\mathrm{PV}$ is present in distinct groups of neurons but its function(s) in these neurons is still unclear. Its capacity to bind calcium ion with high affinity, however, suggests a role in cellular calcium-dependent processes. Since in developing RTN, PV immunoreactivity is found in elongating fibers as well as in cell somata, PV may also help to regulate the synthesis of membrane components, their transport or fusion into developing processes as suggested by Braun et al. (3). This view is further supported by the findings that parvalbumin is able to interact with model vesicles formed with both synthetic and natural phospholipids (16). In RTN, PV-positive fibers seemed to reduce their diameter and immunostaining intensity at $\mathrm{P} 16$. We could not explain this reduction although in the axons of the developing Purkinje cells of zebra finch, more dramatic changes in PV imunoreactivity were reported which related to myelination (3).

GABA immunoreactivity was also found in the RTN of rat embryos with a similar profile to $\mathrm{PV}$. It is also possible that $\mathrm{PV}$ expression is involved in GABAergic transmission in the developing RTN, though there is no information at the present.

This study has shown that the development of the structure and organization of the RTN can be followed using anti-PV antibody as a marker. The next step will be to clarify the relationship between the development of PV staining and innervation with the development of function.

\section{ACKNOWLEDGMENTS}

A. S-O. was supported in Cambridge by the Association to Combat Huntington's Disease (U.K.). This work was supported by the Swiss National Science Foundation (Grant No. 3.139 0.88). The authors are grateful to Dr. Akira Mizutani, the director of our institute for his meaningful discussion and support of this study.

\section{REFERENCES}

1. Aoki, E., Semba, R., Keino, H., Kato-Semba, R. and Kashiwamata, S.: Postnatal development of $\gamma$-aminobutyric acid (GABA) neurons in the cerebellum of jaundiced Gunn rats: A study with anti-GABA antiserum. Biomed. Res. 6; 145-152, 1985.

2. Asanuma, C., Ohkawa, R., Stanfield, B. B. and Cowan, M. W.: Observation on the development of certain ascending inputs to the thalamus in rats. I. Postnatal development. Develop. Brain Res. 41; 159-179, 1988.

3. Braun, K., Schachner, M., Scheich, H. and Heizmann, C. W.: Cellular localization of the $\mathrm{Ca}^{2+}$-binding protein parvalbumin in develoing avian cerebellum. Cell Tissue Res. 243; 69-78, 1986.

4. Celio, M. R. and Heizmann, C. W.: Calcium-binding protein parvalbumin as a neuronal marker. Nature 293; 300-302, 1981.

5. Coggeshall, R. E.: A study of diencephalic development in the albino rat. J. Comp. Neurol. 122; 241-269, 1964. 
6. Heizmann, C. W. and Braun, K.: Calcium binding proteins. Molecular and functional aspects. In the Role of Calcium in Biological Systems, vol. V., ed. by L. J. Anghileri, CRC Press (in press).

7. Heizmann, C. W. and Celio, M. R.: Immunolocalization of parvalbumin. Methods Enzymol. $139 ; 552-570,1987$.

8. Houser, C. R., Vaughn, J. E., Barber, R. P., Roberts, E.: GABA neurons are the major cell type of the nucleus reticularis thalami. Brain Res. 200; 341-354, 1980.

9. Jones, E. G.: Some aspects of the organization of the thalamic reticular complex. J. Comp. Neurol. 162; 285-308, 1975.

10. Kägi, U., Berchtold, M.W. and Heizmann, C. W.: $\mathrm{Ca}^{2+}$-binding protein in rat brain. Characterization, localization and expression during development. J. Biol. Chem. 262; 73147320, 1987.

11. Kawaguchi, Y., Katsumaru, H., Kosaka, T., Heizmann, C. W. and Hama, K.: Fast spiking cells in rat hippocampus ( $\mathrm{CAl}$ region) contain the calcium-binding protein parvalbumin. Brain Res. 416; 369-374, 1987.

12. Lund, R. D. and Mustari, M. J.: Development of the geniculocortical pathway in rats. J. Comp. Neurol. 173; 289-306, 1977.

13. Minderhoud, J. M.: An anatomical study of the efferent connections of the thalamic reticular nucleus. Exp. Brain. Res. 12; 435-446, 1971.

14. Morris, R. J., Beech, J. N. and Heizmann, C. W.: Two distinct phases and mechanisms of axonal growth shown by primary vestibular fibers in the brain. An immunohistochemical study using antibodies to parvalbumin. Neurosci. 27; 597-605, 1988.

15. Ohara, P. T., Sefton, A. J. and Lieberman, A. R.: Mode of termination of afferents from the thalamic reticular nucleus in the dorsal lateral geniculate nucleus of the rat. Brain Res. 197; 503-506, 1980.

16. Permyakov, E. A., Kreimer, D. I., Kalinichenko, L. P., Orlova, A. A. and Shnyrov, V. L.: Interactions of parvalbumins with model phospholipid vesicles. Cell Calcium 10; 71-79, 1989.

17. Peschanski, M., Ralston, H. J. and Roudier, F.: Reticularis thalami afferents to the ventrobasal complex of the rat thalamus: an electron microscope study. Brain Res. 270; 325-329, 1983.

18. Scheibel, M. E. and Scheibel, A. B.: The organization of the nucleus reticularis thalami: a Golgi study. Brain Res. 1; 43-62, 1966.

19. Seto-Ohshima, A., Emson, P. C., Berchtold, M. W. and Heizmann, C. W.: Expression of the parvalbumin gene in the rat brain: a study using hydridization histochemistry. Exp. Brain Res. 75; 653-658, 1989.

20. Steriade, M., Parent, A. and Hada, J.: Thalamic projections of nucleus reticularis thalami of cat: a study using retrograde transport of horseradish peroxidase and fluorescent tracers. $J$. Comp. Neurol. 229; 531-547, 1984. 